\title{
Self-expanding metal stents versus TIPS in treatment of refractory bleeding esophageal varices: a systematic review and meta-analysis
}

다 (우우

\section{Authors}

Babu P. Mohan ${ }^{1}$, Saurabh Chandan ${ }^{2}$, Shahab R. Khan ${ }^{1}$, Rajesh Kotagiri ${ }^{1}$, Lena L. Kassab ${ }^{3}$, Babatunde Olaiya ${ }^{4}$, Suresh Ponnada ${ }^{5}$, Andrew Ofosu ${ }^{6}$, Douglas G. Adler ${ }^{7}$

Institutions

1 Internal Medicine, University of Arizona, Banner University Medical Center, Tucson, Arizona, United States

2 Gastroenterology and Hepatology, University of Nebraska Medical Center, Omaha, Nebraska, United States

3 Internal Medicine, Mayo Clinic, Rochester, Minnesota, United States

4 Internal Medicine, Marshfield Medical Center, Marshfield, Wisconsin, United States

5 Internal Medicine, Carilion Roanoke Memorial Hospital, Roanoke, Virginia, United States

6 Division of Gastroenterology and Hepatology, The Brooklyn Hospital Center, Brooklyn, New York, United States

7 Division of Gastroenterology and Hepatology, University of Utah School of Medicine, Salt Lake City, Utah, United States

submitted 6.8.2019

accepted after revision $\quad$ 15.10.2019

\section{Bibliography}

DOI https://doi.org/10.1055/a-1067-4563 |

Endoscopy International Open 2020; 08: E291-E300

(c) Georg Thieme Verlag KG Stuttgart · New York elSSN 2196-9736

\section{Corresponding author}

Douglas G. Adler MD, FACG, AGAF, FASGE, Professor of Medicine, Director of Therapeutic Endoscopy, Director, GI fellowship Program, Gastroenterology and Hepatology, University of Utah School of Medicine, Huntsman Cancer Center, 30N 1900E 4R118, Salt Lake City, Utah 84132

Fax: +1-801-581-8007

Douglas.adler@hsc.utah.edu $\bigoplus$ Supplementary materia

Online content viewable at:

https://doi.org/10.1055/a-1067-4563

\section{ABSTRACT}

Background and study aims Refractory and recurrent esophageal variceal (EV) bleeding can be life threatening. Self-expanding metal stents (SEMS) have been used as a "bridge" therapy. However, their role in the treatment protocol is not established due to paucity in data.

Methods We searched multiple databases from inception through May 2019 to identify studies that reported on SEMS and TIPS in refractory EV hemorrhage. Our primary goals were to analyze and compare the pooled all-cause mortality, immediate bleeding control and rebleeding rates.

Results Five hundred forty-seven patients from 21 studies were analyzed (SEMS: 12 studies, 176 patients; TIPS: 9 studies, 398 patients). The pooled rate of all-cause mortality with SEMS was $43.6 \%\left(95 \% \mathrm{Cl} 28.6-59.8, \mathrm{I}^{2}=38\right)$ and with TIPS was $27.9 \%\left(95 \% \mathrm{Cl} 16.3-43.6, \mathrm{I}^{2}=91\right)$. The pooled rate of immediate bleeding control with SEMS was $84.5 \%$ (95\% $\left.\mathrm{Cl} 74-91.2, \mathrm{I}^{2}=40\right)$ and with TIPS was $97.9 \%$ (95\% Cl 87.7$\left.99.7, \mathrm{I}^{2}=0\right)$. The pooled rate of rebleeding with SEMS was $19.4 \%\left(95 \% \mathrm{Cl} 11.9-30.4, \mathrm{I}^{2}=32\right)$ and with TIPS was $8.8 \%$ (95\% Cl 4.8-15.7, $\left.\mathrm{I}^{2}=40\right)$.

Conclusion Use of SEMS in refractory EV hemorrhage demonstrates acceptable immediate bleeding control with good technical success rate. Mortality and rebleeding rates were lesser with TIPS, however, its superiority and/ or inferiority cannot be validated due to limitations in the comparison methodology. 


\section{Introduction}

Esophageal variceal (EV) bleeding is one of the most life-threatening complications of portal hypertension, with mortality rates of $15 \%$ to $20 \%$ [1]. The current recommendation is to hemodynamically stabilize the patient and promptly initiate vasoactive drugs like terlipressin and/or somatostatin analogues, followed by endoscopic ligation of EV, ideally within 12 hours of presentation [2,3]. However, $20 \%$ to $30 \%$ of patients can rebleed and become refractory to standard treatment [4].

In refractory patients, treatment options include surgical and/or non-surgical creation of a porto-systemic shunt that reduces the portal pressure and controlling the bleeding by tamponade. Surgical options of shunt creation are as follows: portocaval (portal vein and vena cava), mesocaval (mesenteric vein and vena cava), spleno-renal shunt (proximal splenic vein and left renal vein), and externally reinforced shunts that can be either mesocaval or portocaval. Non-surgical options include balloon tamponade, trans-jugular intra-hepatic portosystemic shunt (TIPS) that is created by an interventional radiologist under fluoroscopic guidance and the placement of a self-expanding metal stent (SEMS) [2,3].

Balloon tamponade was the most commonly used option in the past. However, it has fallen out of favor due to risk of pressure-induced necrosis of the esophagus and to the fact that it can only be used for, at most, 24-48 hours [5]. Use of TIPS is limited by technical difficulties and availability [6]. Studies published thus far report the use of SEMS as a 'bridge' therapy with majority of the patients eventually being treated with EV banding and/ or TIPS [3, 7-9]. Although the current Baveno consensus workshop recommends the use of SEMS, an evidence-based approach to guide the use of SEMS in potentially life-threatening refractory EV bleeding is not established.

The goals of this meta-analysis were to study the clinical outcomes of SEMS, and use the clinical outcomes of TIPS as a comparator, in refractory EV hemorrhage.

\section{Methods}

\section{Search strategy}

We conducted a comprehensive search of several databases from inception to May 24, 2019. The databases included Ovid MEDLINE $®$ and Epub Ahead of Print, In-Process and other nonindexed citations, Ovid Embase, Ovid Cochrane Central Register of Controlled trials, Ovid Cochrane Database of Systematic Reviews, and Scopus. An experienced medical librarian using inputs from the study authors helped with the literature search. Controlled vocabulary supplemented with keywords was used to search for studies of interest. The full search strategy is available in Appendix 1. The PRISMA and MOOSE checklist were followed and are provided in Appendix 2 and $3[10,11]$.

\section{Study selection}

In this meta-analysis, we included studies that evaluated clinical outcomes of SEMS and studies that evaluated outcomes of TIPS in patients with refractory EV hemorrhage. Studies were included irrespective of the study sample-size, inpatient/out- patient setting, and geography as long as they provided data needed for the analysis.

Studies done in the pediatric population (age <18 years), and studies not published in English language were our only exclusion criteria. In case of multiple publications from the same cohort and/or overlapping cohorts, data from the most recent and/or most appropriate comprehensive report were retained.

\section{Data abstraction and quality assessment}

Data on study-related outcomes in individual studies were abstracted onto a standardized form by at least two authors (SRK, RK), and two authors (BPM, SC) did the quality scoring independently. Primary study authors were contacted via email as needed for further information and/or clarification on data.

The Newcastle-Ottawa scale for cohort studies was used to assess the quality of studies [12]. This quality score consisted of 8 questions, the details of which are provided in Supplementary Table 1.

\section{Outcomes assessed}

1. Pooled rate of all-cause mortality,

2. Pooled rate of immediate EV bleeding control,

3. Pooled rate of rebleeding, and

4. Pooled rate of adverse events.

Comparison analysis: The outcomes with SEMS were compared with the outcomes of TIPS.

\section{Assessment methodology and definitions}

The collected data was matched between the groups (SEMS and TIPS) before statistical analysis. Although, this model of comparison is indirect, the approach is comparable to a retrospective case-control study with matched groups [13]. Refractory EV bleeding was defined according to the Baveno IV and V guidelines: fresh hematemesis or aspiration of $>100 \mathrm{ml}$ of fresh blood via the nasogastric tube beyond 2 hours after endoscopy and/or a drop of $3 \mathrm{~g} / \mathrm{dL}$ in hemoglobin without blood transfusion $[5,14]$.

Rebleeding was defined as per the Baveno $V$ guidelines: evidence of rebleeding from portal hypertensive sources (hematemesis, malena, aspiration of $>100 \mathrm{~mL}$ of fresh blood via the nasogastric tube beyond two hours after endoscopy and/or a drop of $3 \mathrm{~g} / \mathrm{dL}$ in hemoglobin without blood transfusion [14].

\section{Statistical analysis}

We used meta-analysis techniques to calculate the pooled estimates in each case following the methods suggested by DerSimonian and Laird using the random-effects model [15]. When incidence of an outcome was zero in a study, a continuity correction of 0.5 was added to the number of incident cases before statistical analysis [16]. We assessed heterogeneity between study-specific estimates by using Cochran Q statistical test for heterogeneity, 95\% prediction interval (PI), which deals with the dispersion of the effects [17-19]. and the $\mathrm{I}^{2}$ statistics [20, 21 ]. In this, values $<30 \%, 30 \%$ to $60 \%, 61 \%$ to $75 \%$, and $>75 \%$ were suggestive of low, moderate, substantial, and considerable heterogeneity, respectively [22]. Publication bias was as- 


\begin{tabular}{|c|c|c|c|c|c|c|c|c|c|}
\hline 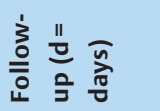 & 8 & $\grave{c}$ & 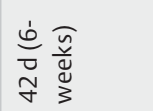 & $\underset{\mathscr{\gamma}}{\tilde{y}}$ & 品 & 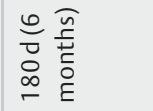 & $\stackrel{\widetilde{Y}}{\mathscr{\gamma}}$ & 品 & 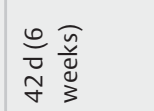 \\
\hline 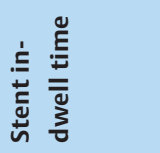 & 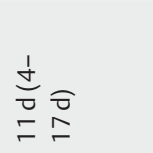 & 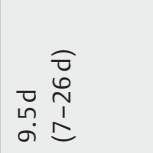 & 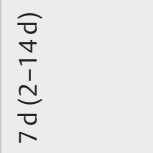 & 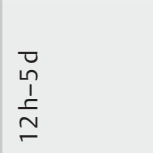 & 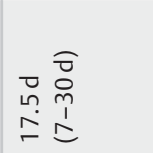 & 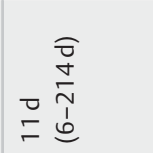 & 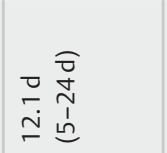 & $\grave{E}$ & 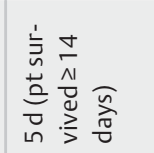 \\
\hline 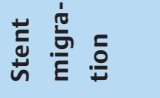 & 0 & $\stackrel{m}{i}$ & $\wedge$ & $\sim$ & 0 & - & $r$ & in & $\stackrel{m}{\sim}$ \\
\hline 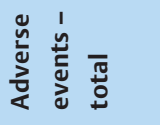 & 0 & $\bar{E}$ & 0 & $\sim$ & 0 & - & $\sigma$ & 乞n & 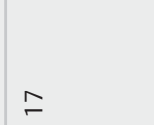 \\
\hline 言竞方高 & 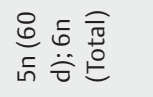 & $r$ & $E \stackrel{\widehat{\widetilde{I}}}{\stackrel{I}{ \pm}}$ & $\stackrel{\widehat{\widetilde{N}}}{\stackrel{\sim}{ \pm}}$ & in & $m$ & $m \stackrel{\widehat{̃}}{\stackrel{I}{ \pm}}$ & $m$ & $\tilde{N}$ \\
\hline 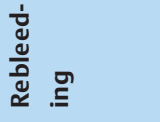 & $m$ & $\grave{E}$ & 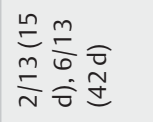 & 0 & - & - & - & - & $\approx$ \\
\hline 竞总范 & $\begin{array}{l}\dot{0} \\
\dot{\phi} \\
\dot{\ddot{x}} \\
\ddot{\sim} \dot{U}\end{array}$ & $\grave{E}$ & $\stackrel{m}{\ddot{x}} \stackrel{0}{\ddot{\dot{\varphi}}} \stackrel{0}{\dot{U}}$ & 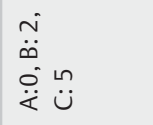 & $\grave{E}$ & $\begin{array}{l}\overline{\dot{\theta}} \\
\dot{\ddot{x}} \overline{\dot{u}}\end{array}$ & $\begin{array}{l}\stackrel{\varphi}{\dot{\omega}} \\
\check{\ddot{x}} \ddot{\vartheta}\end{array}$ & $\bar{E}$ & 妾 \\
\hline$\frac{\pi}{\tilde{N}}$ & 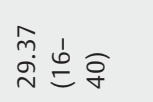 & $\grave{c}$ & 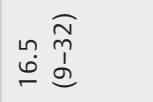 & $\lesssim \stackrel{1}{\approx} \widetilde{m}$ & 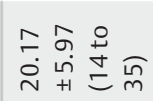 & $\bar{\sim} \equiv \stackrel{\frac{1}{\infty}}{\sim}$ & 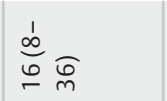 & $\grave{c}$ & 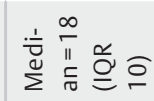 \\
\hline$\frac{u}{\Sigma}$ & $\frac{N}{6}$ & $\stackrel{\hbar}{n}$ & $\stackrel{o}{m}$ & $\frac{n}{n}$ & $\stackrel{\equiv}{\equiv}$ & $\frac{N}{m}$ & $\frac{m}{\infty}$ & $\frac{\theta}{\infty}$ & $\stackrel{0}{\infty}$ \\
\hline 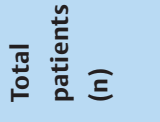 & $\infty$ & 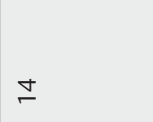 & $\stackrel{m}{r}$ & $r$ & $\simeq$ & Ln & $\mp$ & $\simeq$ & $\stackrel{m}{m}$ \\
\hline 离 & $\stackrel{\infty}{\tilde{\varphi}} \bar{\Xi}$ & 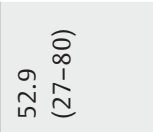 & 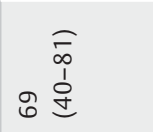 & 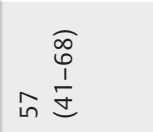 & 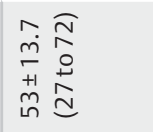 & 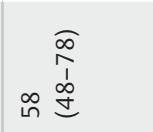 & 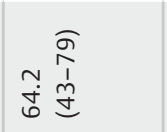 & 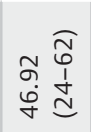 & 号芒 \\
\hline 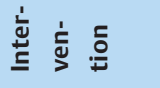 & $\sum_{\text {出 }}^{n}$ & $\sum_{\text {出 }}^{n}$ & $\sum_{\text {出 }}^{n}$ & $\sum_{\text {出 }}^{n}$ & $\sum_{\text {出 }}^{n}$ & $\sum_{\tilde{W}}^{n}$ & $\sum_{\tilde{U}}^{n}$ & $\sum_{\tilde{H}}^{n}$ & $\sum_{\tilde{U}}^{n}$ \\
\hline 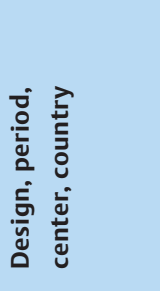 & 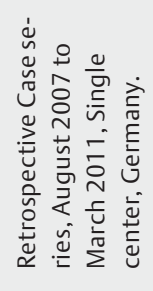 & 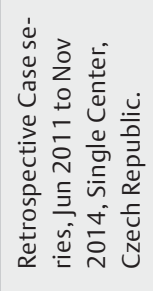 & 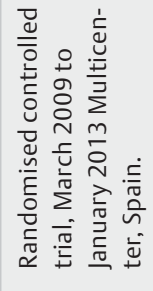 & 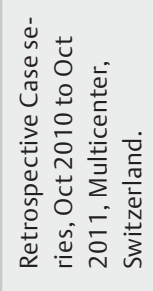 & 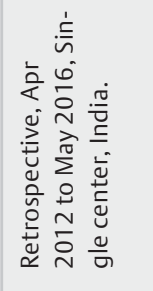 & 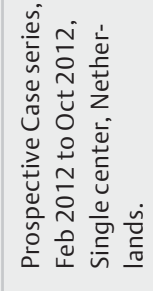 & 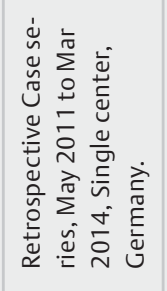 & 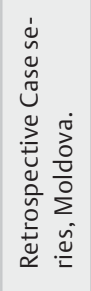 & 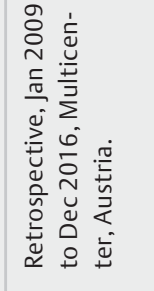 \\
\hline 胥 & 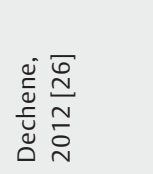 & 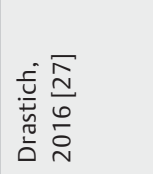 & 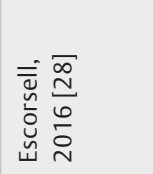 & 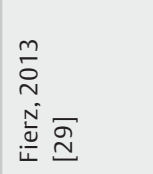 & 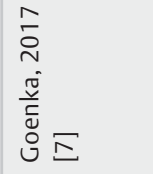 & 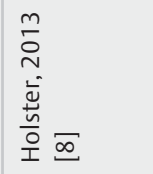 & 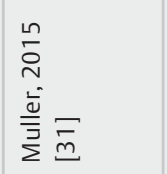 & 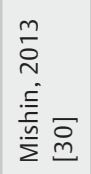 & 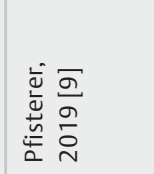 \\
\hline
\end{tabular}




\begin{tabular}{|c|c|c|c|c|c|c|c|c|c|c|}
\hline 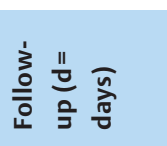 & $\underset{\mathscr{J}}{\tilde{y}}$ & $\grave{E}$ & 8 & 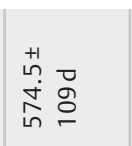 & 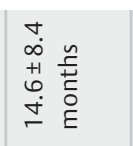 & 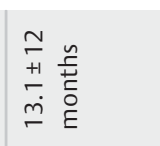 & 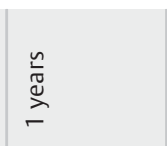 & 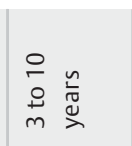 & 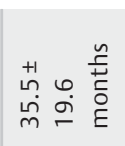 & 点 \\
\hline 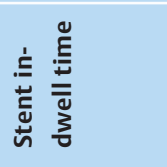 & 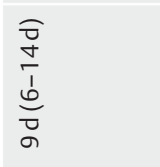 & $\underset{\substack{J \\
⿱ 亠}}{\sim}$ & 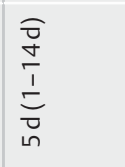 & $\grave{c}$ & $\grave{c}$ & $\grave{E}$ & $\bar{E}$ & $\grave{c}$ & $\grave{c}$ & $\grave{c}$ \\
\hline 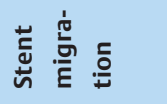 & 0 & 0 & $r$ & È & $\grave{E}$ & $\bar{E}$ & $\bar{E}$ & $\bar{E}$ & $\bar{E}$ & $\grave{E}$ \\
\hline 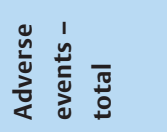 & - & $r$ & $\infty$ & $\grave{E}$ & $\grave{E}$ & $\grave{c}$ & $\grave{E}$ & $\grave{c}$ & $\stackrel{\varphi}{\circ}$ & $\stackrel{\nabla}{\sim}$ \\
\hline 市竞言 & 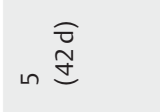 & $\sigma$ & $\circ \stackrel{\widehat{0}}{\circ}$ & 들 & $\nabla$ & 0 & $\infty$ & 8 & $\stackrel{m}{r}$ & $a$ \\
\hline 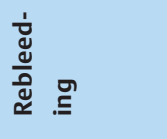 & $m$ & $\sim$ & 0 & $m$ & 0 & $\sim$ & $m$ & $\bar{c}$ & $m$ & 0 \\
\hline 竞䍃范 & $\grave{E}$ & $\begin{array}{l}\dot{\infty} \\
\dot{0} \\
\stackrel{\dot{x}}{\dot{u}} \\
\dot{U}\end{array}$ & 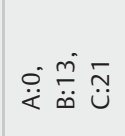 & $\grave{E}$ & 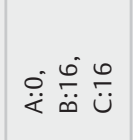 & $\stackrel{0}{\dot{x}} \stackrel{\infty}{\check{\theta}} \stackrel{\tilde{U}}{\dot{U}}$ & 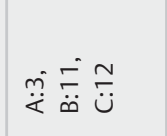 & 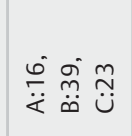 & 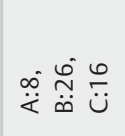 & $\begin{array}{l}\tilde{\dot{\theta}} \\
\stackrel{\dot{u}}{\dot{x}}\end{array}$ \\
\hline$\frac{\pi}{\sum^{\circ}}$ & $\approx \stackrel{1}{\tilde{n}} \widehat{\bar{m}}$ & $\grave{E}$ & $\grave{E}$ & $\grave{E}$ & $\begin{array}{l}\stackrel{+}{1} \\
\stackrel{\sim}{n} \\
\stackrel{n}{n} \text { n }\end{array}$ & 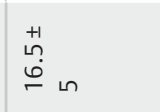 & $\grave{c}$ & $\grave{c}$ & $\grave{E}$ & $\begin{array}{l}+1 \\
\text { aे } \\
\text { iे g. }\end{array}$ \\
\hline$\frac{\omega}{\Sigma}$ & $\bar{\sigma}$ & $\frac{7}{n}$ & $\frac{\bar{m}}{m}$ & $\frac{n}{\sigma}$ & $\frac{\Sigma}{\bar{i}}$ & $\frac{\bar{F}}{\bar{f}}$ & $\frac{\sqrt{n}}{\frac{a}{4}}$ & $\frac{\substack{\frac{N}{0} \\
\text { in }}}{2}$ & $\frac{\bar{d}}{\frac{\pi}{2}}$ & 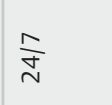 \\
\hline 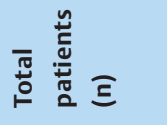 & $\stackrel{\circ}{\circ}$ & $\stackrel{6}{\circ}$ & 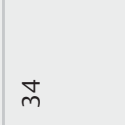 & $\stackrel{\Xi}{\sim}$ & $\tilde{m}$ & ケ̊ & $\stackrel{\mathscr{N}}{\sim}$ & $\stackrel{\infty}{\wedge}$ & in & $\bar{m}$ \\
\hline 离 & 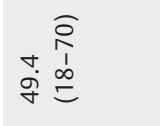 & 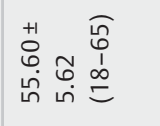 & 京 & $\begin{array}{l}\infty \\
\substack{\infty \\
\dot{d}} \\
\stackrel{d}{\stackrel{d}{d}}\end{array}$ & $\begin{array}{l}\stackrel{0}{+1} \\
\text { in } \\
\text { n }\end{array}$ & 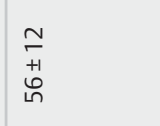 & 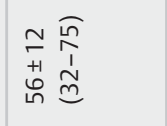 & 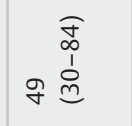 & $\begin{array}{l}+1 \\
\stackrel{\sim}{\sim} \stackrel{\sim}{m} \\
\end{array}$ & 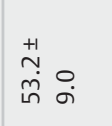 \\
\hline 咅 & $\sum_{\tilde{\omega}}^{n}$ & $\sum_{\tilde{\omega}}^{n}$ & $\sum_{u 山}^{n}$ & $\stackrel{\varrho}{\models}$ & $\cong$ & $\stackrel{\cong}{\models}$ & $\cong$ & $\stackrel{\varrho}{\risingdotseq}$ & $\stackrel{\breve{~}}{F}$ & $\stackrel{\check{\varrho}}{F}$ \\
\hline 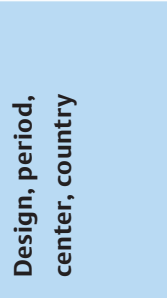 & 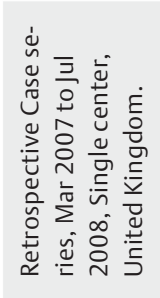 & 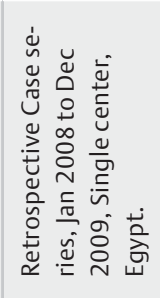 & 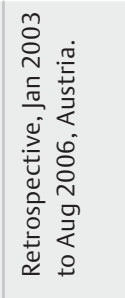 & 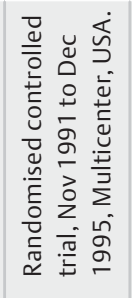 & 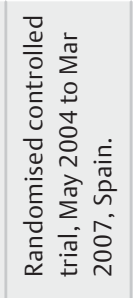 & 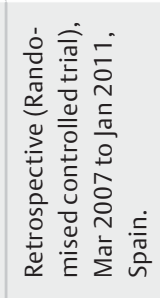 & 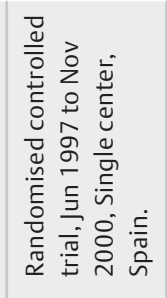 & 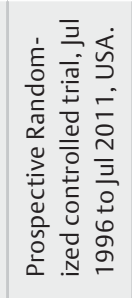 & 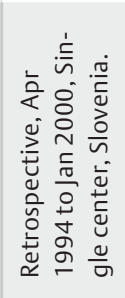 & 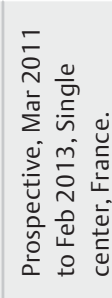 \\
\hline 离 & 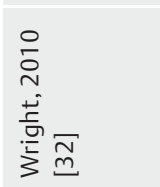 & 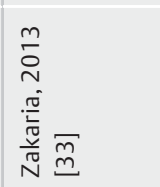 & 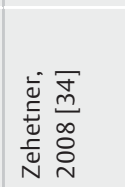 & 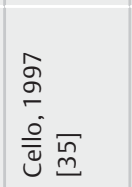 & 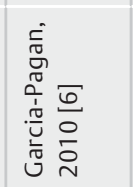 & 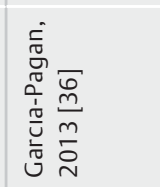 & 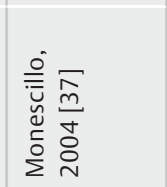 & $\begin{array}{l}\stackrel{N}{5} \\
\text { N } \\
\text { 递 } \\
\text { 은 }\end{array}$ & 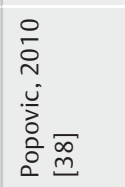 & 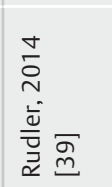 \\
\hline
\end{tabular}




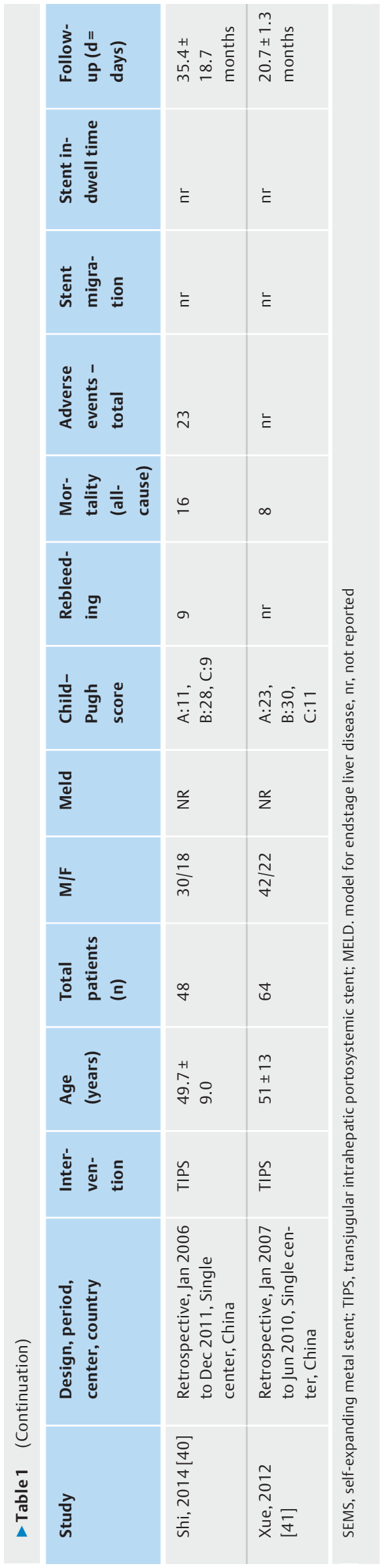

certained, qualitatively, by visual inspection of funnel plot and quantitatively, by the Egger test [23]. When publication bias was present, further statistics using the fail-Safe $\mathrm{N}$ test and Duval and Tweedie's "Trim and Fill" test was used to ascertain the impact of the bias [24]. Three levels of impact were reported based on the concordance between the reported results and the actual estimate if there were no bias. The impact was reported as minimal if both versions were estimated to be same, modest if effect size changed substantially but the final finding would still remain the same, and severe if basic final conclusion of the analysis is threatened by the bias [25].

All analyses were performed using Comprehensive Meta-Analysis (CMA) software, version 3 (BioStat, Englewood, New Jersey, United States).

\section{Results}

\section{Search results and population characteristics}

From an initial 395 studies, 214 records were screened and 33 full-length articles were assessed. Twenty-one studies were included in the final analysis, of which 12 studies reported on the outcomes with SEMS [7-9,26-34] and 9 reported on the outcomes with TIPS [6, 35-42].

The schematic diagram of study selection is illustrated in Supplementary Fig. 1. Two SEMS studies had cohort overlap and the most comprehensive one was retained for the analysis (Wright 2010 with Hogan 2009 and Zehetner 2008 with Hubmann 2006) [32, 34, 43, 44].

Baseline population characteristics were comparable between the SEMS and TIPS groups. The mean and/or median age ranged from 46 years to 69 years, with a predominantly male population (74\%). Twelve percent of the patients were Child's A, 39\% were Child's B and the rest were Child's C cirrhotics. The mean MELD score ranged from 16 to 29.37 in SEMS group and 15.5 to 20.9 in TIPS groups. The ELLA-CS stent (SXELLA Stent Danis, Hradec Kralove, Czech Republic) was used in all SEMS studies with a stent indwell time ranging from 1 to 30 days. Data was not available on the severity of the bleeding and the interventional procedure was done on an emergency basis. The population characteristics are described in > Table 1.

\section{Characteristics and quality of included studies}

One SEMS study was prospective, whereas rest were retrospective [8]. Three were from multicenter data [9, 28, 29]. No studies were population-based. Overall, four studies were considered high quality and the rest were medium quality $[9,28,29$, 34]. There were no low-quality studies. All TIPS studies in the comparator group were considered high quality. The detailed assessment of study quality can be found in Supplementary Table 1

\section{Meta-analysis outcomes}

A total of 574 patients were included in the analysis from 21 studies. 176 patients were treated with SEMS in 12 studies and 398 patients were treated with TIPS in 9 studies.

The pooled rate of all-cause mortality with SEMS was $43.6 \%$ (95\% Cl 28.6-59.8) and with TIPS was $27.9 \%(95 \% \mathrm{Cl} 16.3-$ 


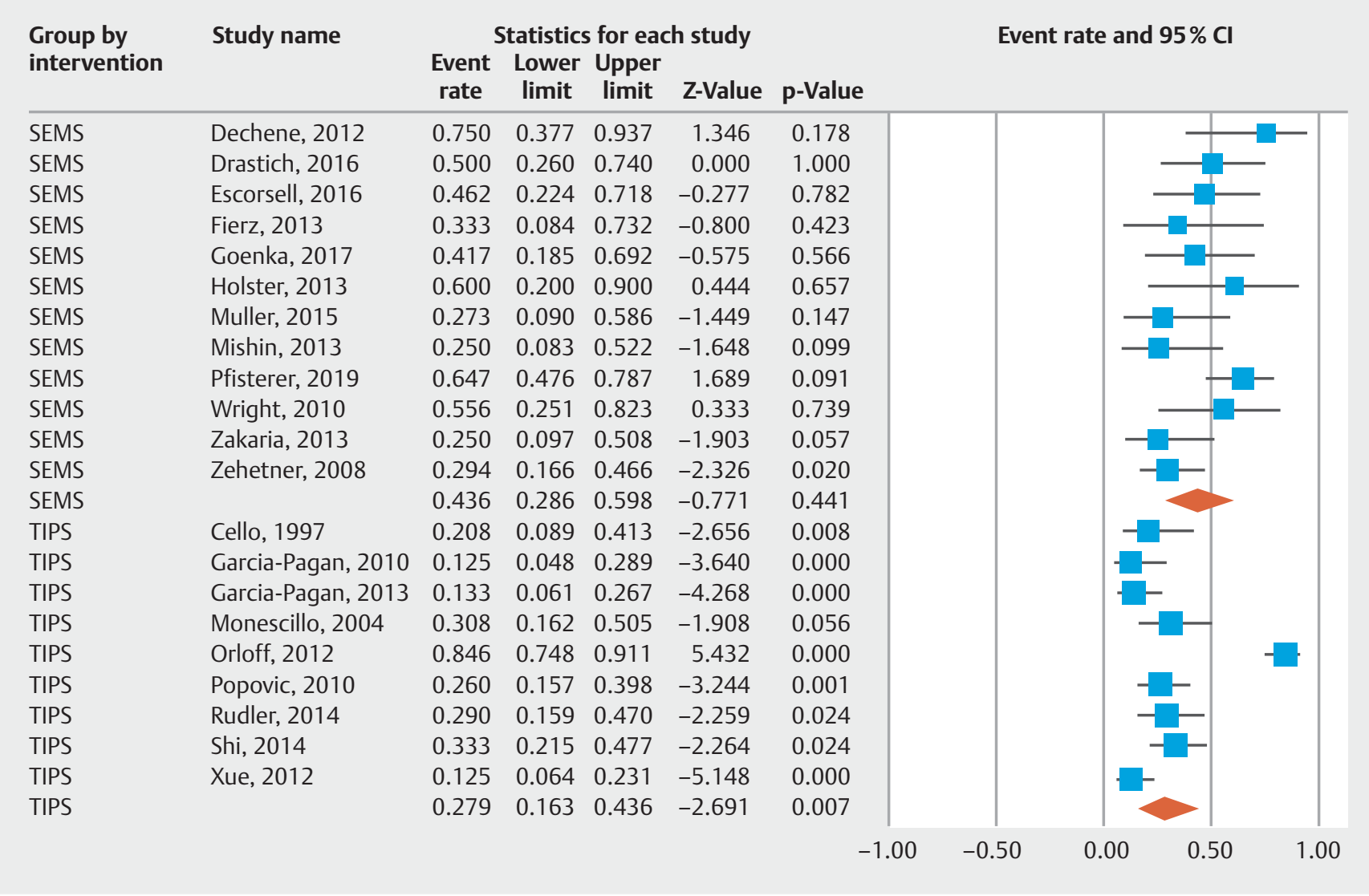

Fig. 1 Forest plot, mortality.

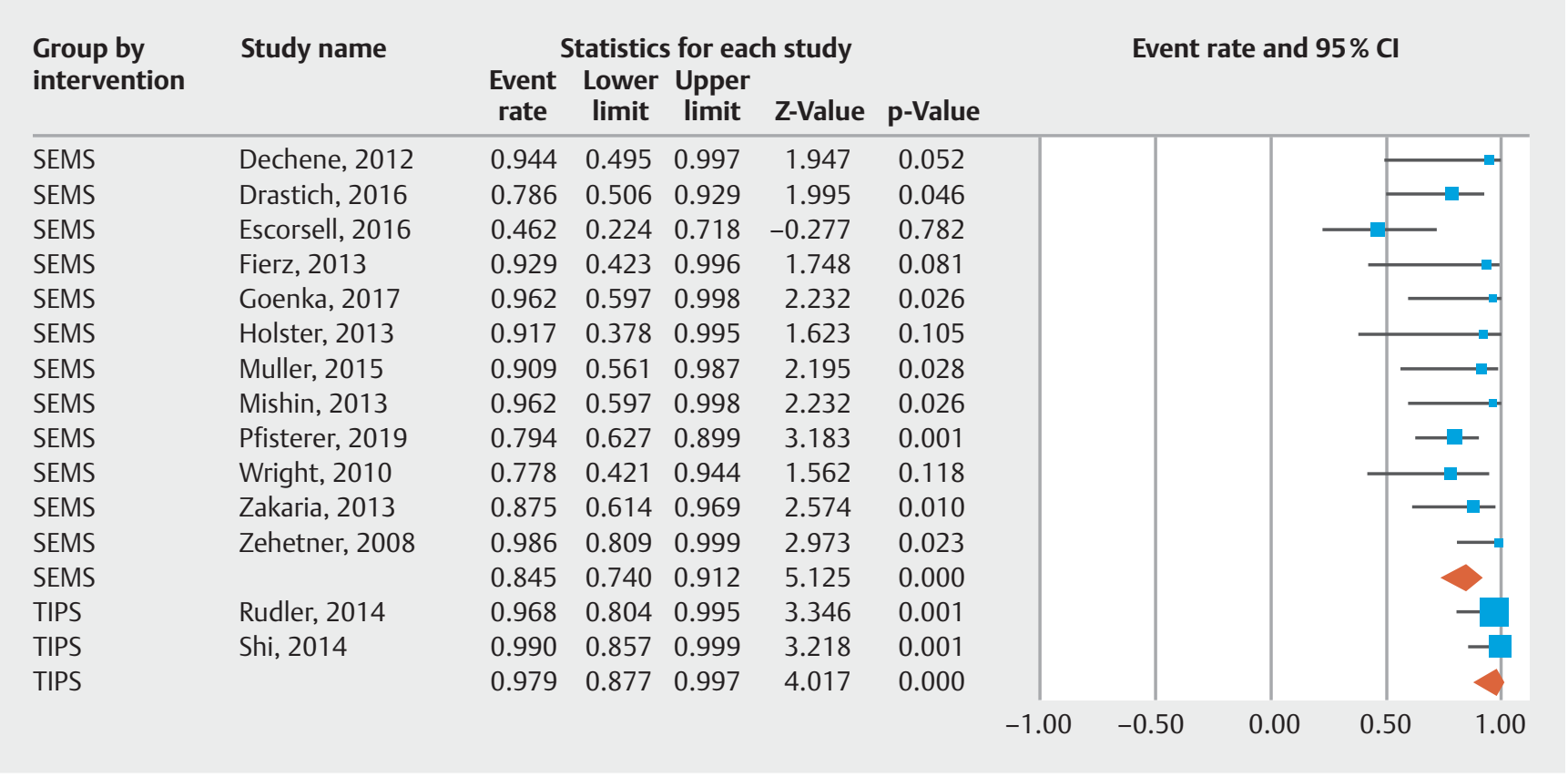

- Fig. 2 Forest plot, immediate bleeding control.

43.6) ( Fig. 1). The pooled rate of immediate bleeding control with SEMS was $84.5 \%(95 \% \mathrm{Cl} 74-91.2)$ and with TIPS was $97.9 \%$ (95\% Cl 87.7-99.7) (\Fig.2). The pooled rate of re- bleeding with SEMS was $19.4 \%(95 \% \mathrm{Cl} 11.9-30.4)$ and with TIPS was $8.8 \%$ (95\% Cl 4.8-15.7) (> Fig. 3). 


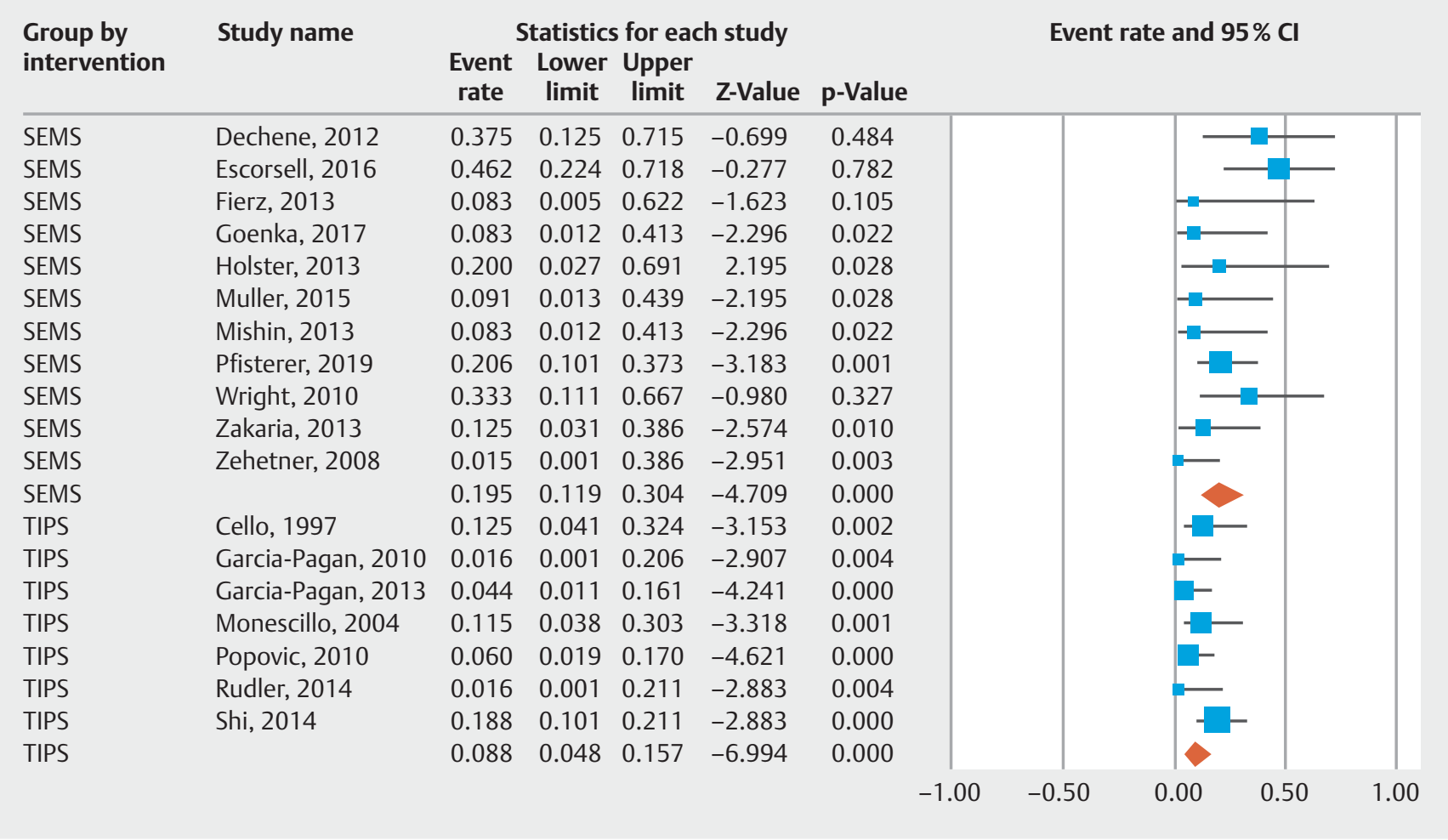

- Fig. 3 Forest plot, rebleeding.

- Table 2 Summary of pooled rates.

\begin{tabular}{|c|c|c|}
\hline Outcomes & SEMS & TIPS \\
\hline \multicolumn{3}{|c|}{$\begin{array}{l}\text { Pooled rate ( } 95 \% \text { confidence interval) } \\
95 \% \text { Prediction interval (PI), } 1^{2} \text { values }\end{array}$} \\
\hline Mortality & $\begin{array}{l}43.6 \%(28.6-59.8) \\
\text { PI: } 18 \text { to } 73,38\end{array}$ & $\begin{array}{l}27.9 \%(16.3-43.6) \\
\text { PI: } 2 \text { to } 88,91\end{array}$ \\
\hline Immediate bleeding control & $\begin{array}{l}84.5 \%(74-91.2) \\
\text { PI: } 50 \text { to } 97,40\end{array}$ & $\begin{array}{l}97.9 \% \text { ( } 87.7-99.7) \\
\text { PI: NA (due to limited number of studies) }\end{array}$ \\
\hline Rebleeding & $\begin{array}{l}19.4 \%(11.9-30.4) \\
\text { PI: } 6 \text { to } 50,32\end{array}$ & $\begin{array}{l}8.8 \%(4.8-15.7) \\
\mathrm{PI}: 2 \text { to } 33,40\end{array}$ \\
\hline Technical success & $\begin{array}{l}88.3 \%(81.7-92.7) \\
\text { PI: } 80 \text { to } 93,0\end{array}$ & $\begin{array}{l}91 \%(86.2-94.2) \\
\text { PI: } 73 \text { to } 97,26\end{array}$ \\
\hline All adverse events & $\begin{array}{l}36.9 \%(26-49.2) \\
\text { PI: } 11 \text { to } 74,52\end{array}$ & $\begin{array}{l}41.4 \%(26.5-58.1) \\
\text { PI: } 0.4 \text { to } 99,29\end{array}$ \\
\hline Stent migration & $\begin{array}{l}31.8 \%(22-43.5) \\
\text { PI: } 11 \text { to } 63,41\end{array}$ & NA \\
\hline
\end{tabular}

The pooled rate of technical success with SEMS was $88.3 \%$ (95\% Cl 81.7-92.7) and with TIPS was $91 \%$ (95\% CI 86.294.2). The pooled rate of all adverse events with SEMS was $36.9 \%(95 \% \mathrm{Cl} 26-49.2)$ and with TIPS was $41.4 \%(95 \% \mathrm{Cl}$ 26.5-58.1). The pooled rate of stent migration was $31.8 \%$ (95\% Cl 22-43.5). (Supplementary Fig. 2, 3 and 4) The pooled results are summarized in $>$ Table 2 .

\section{Validation of meta-analysis results}

\section{Sensitivity analysis}

To assess whether any one study had a dominant effect on the meta-analysis, we excluded one study at a time and analyzed its effect on the main summary estimate. On this analysis, no single study significantly affected the outcome or the heterogeneity. 


\section{Heterogeneity}

We assessed dispersion of the calculated rates using the prediction interval $(\mathrm{PI})$ and $\mathrm{I}^{2}$ percentage values. The $\mathrm{PI}$ gives an idea of the range of the dispersion and $\mathrm{I}^{2}$ tell us what proportion of the dispersion is true vs chance [19]. The calculated PIs and corresponding $\mathrm{I}^{2}$ values are reported in $>$ Table 2 . The calculated PI was wide except for the pooled rates of technical success. However, the $\mathrm{I}^{2}$ heterogeneity was mild to moderate except for the morality rate with TIPS. This means that the reported pooled clinical outcomes may or may not be valid to the real-world scenario.

\section{Publication bias}

Based on visual inspection of the funnel plot as well as quantitative measurement that used the Egger regression test, there was evidence of publication bias (supplementary figure 5 , Eggers 2-talied $P=0.04$ ). Further statistics using the fail-Safe $N$ test and Duval and Tweedie's "Trim and Fill" test revealed that the impact of the possible publication bias appeared to be minimal and would not change the calculated estimate or the conclusion of this meta-analysis.

\section{Quality of evidence}

The quality of evidence was rated for results from the meta-analysis according to the GRADE working group approach [45]. Observational studies begin with a low-quality rating and based on the risk of bias, indirectness, heterogeneity, and publication bias, the quality of this meta-analysis would be considered as low-quality evidence.

\section{Discussion}

Our study demonstrates that use of SEMS is associated with a pooled all-cause mortality rate of $44 \%$, immediate bleeding control rate of $85 \%$ and a rebleeding rate of $19 \%$. A recent multicenter study by Pfiesterer et al reported a mortality rate of $47 \%$ with the use of SEMS in refractory EV bleeding and our results are on par with this study [9]. We report a pooled allcause mortality rate of $28 \%$ with TIPS. The mortality rates with SEMS and TIPS seemed comparable.

The pooled immediate bleeding control and rebleeding rates are the key findings of this study. $85 \%$ of patients achieved immediate bleeding control with SEMS, whereas with TIPS $98 \%$ of patients achieved immediate bleeding control. The pooled rebleeding rate with SEMS was $19 \%$ and with TIPS was $9 \%$. Based on our comparison method, the probability that the proportion of patients undergoing TIPS having a successful outcome seemed to be more than the ones having a SEMS placed. The pooled rate of technical success with SEMS was $88 \%$ and with TIPS was $91 \%$. Although the technical success rates were comparable, the prompt availability of emergent TIPS continues to be an issue at many centers due to limited resources and experienced personnel.

The Baveno VI recommendation on the use of SEMS in refractory EV hemorrhage is based on its favorable safety profile when compared to balloon-tamponade [3]. Our analysis of the adverse events with SEMS revealed a pooled rate of $37 \%$ and was comparable to the pooled adverse event rate with TIPS, which was $41 \%$. Stent migration is a significant problem and our analysis revealed that the stent migrated in approximately one-third of the patients ( $31 \%$ ).

How does our study compare to other published reviews? The meta-analysis by McCarty and Njei reported a pooled bleeding control rate of $96 \%$ [46]. The pooled outcomes reported in that study is of questionable validity due to the inclusion of studies that had overlapping cohorts (Hubmann 2006 with Zehetner 2008 and Hogan 2009 with Wright 2010) [32, $34,43,44]$. Another meta-analysis by Marot et al., had similar limitation and only reported the mortality and adverse events [47]. Our study, on the contrary, has avoided studies with overlapping cohorts and we have presented the pooled results in perspective to the pooled outcomes of TIPS in refractory EV bleeding, thereby enabling a side-by-side comparison. The meta-analysis by Shao et al., report that SEMS may be considered in patients with EV bleeding refractory to conventional therapy and their pooled rates are comparable to this study [48]. The meta-analysis by Qi eta I on TIPS in acute EV bleeding reported that TIPS with covered stents might improve the overall survival of high-risk patients with acute EV bleeding [49]. The results are comparable to the pooled mortality rates reported in this study.

The strengths of this review are as follows: systematic literature search with well-defined inclusion criteria, careful exclusion of redundant studies, inclusion of good quality studies with detailed extraction of data and rigorous evaluation of study quality. Our pooled rates are calculated from $176 \mathrm{pa}$ tients treated with SEMS and 398 patients treated with TIPS. There are limitations to this study, most of which are inherent to any meta-analysis. The included studies were not entirely representative of the general population and community practice, with most studies being performed in tertiary-care referral centers. Our analysis had studies that were retrospective in nature contributing to selection bias. It is practically impossible to compare SEMS to TIPS in refractory EV bleeding by RCT methods and a network meta-analysis is not possible due to lack of studies with a common comparator. Our study presents the results of SEMS and TIPS side by side, however, our analysis has the limitation of retrospective comparison and therefore we do not comment on the superiority and/ or inferiority of one modality to other. Nevertheless, our study is the best available estimate in literature thus far with respect to the clinical outcomes of SEMS and TIPS in refractory EV bleeding.

\section{Conclusion}

In conclusion, based on our meta-analysis, the use of SEMS in refractory EV bleeding demonstrates acceptable technical success and immediate bleeding control. However, the pooled mortality rate and rebleeding rate with TIPS seem to be lesser than SEMS. We, unfortunately, are unable to validate the results of comparison between the two modalities due to the limitations in our retrospective comparison methodology. 


\section{Competing interests}

The authors declare that they have no conflict of interest.

\section{References}

[1] Garcia-Pagan JC, Reverter E, Abraldes JG et al. Acute variceal bleeding. Semin Respir Crit Care Med 2012; 33: 46-54

[2] Reiberger T, Puspok A, Schoder M et al. Austrian consensus guidelines on the management and treatment of portal hypertension (Billroth III). Wien Klin Wochenschr 2017; 129: 135-158

[3] de Franchis R. Expanding consensus in portal hypertension: Report of the Baveno VI Consensus Workshop: Stratifying risk and individualizing care for portal hypertension. J Hepatol 2015; 63: 743-752

[4] Sharara AI, Rockey DC. Gastroesophageal variceal hemorrhage. N Engl J Med 2001; 345: 669-681

[5] de Franchis R. Evolving consensus in portal hypertension. Report of the Baveno IV consensus workshop on methodology of diagnosis and therapy in portal hypertension. J Hepatol 2005; 43: 167-176

[6] Garcia-Pagan JC, Caca K, Bureau C et al. Early use of TIPS in patients with cirrhosis and variceal bleeding. N Engl J Med 2010; 362: 2370 2379

[7] Goenka MK, Goenka U, Tiwary IK et al. Use of self-expanding metal stents for difficult variceal bleed. Indian J Gastroenterol 2017; 36: 468-473

[8] Holster IL, Kuipers EJ, Van Buuren HR et al. Self-expandable metal stents as definitive treatment for esophageal variceal bleeding. Endoscopy 2013; 45: 485-488

[9] Pfisterer N, Riedl F, Pachofszky T et al. Outcomes after placement of a SX-ELLA oesophageal stent for refractory variceal bleeding-A national multicentre study. Liver Int 2019; 39: 290-298

[10] Moher D, Liberati A, Tetzlaff J et al. Preferred reporting items for systematic reviews and meta-analyses: The prisma statement. Annals Internal Med 2009; 151: 264-269

[11] Stroup DF, Berlin JA, Morton SC et al. Meta-analysis of observational studies in epidemiology: a proposal for reporting. Meta-analysis Of Observational Studies in Epidemiology (MOOSE) group. JAMA 2000; 283: 2008-2012

[12] Stang A. Critical evaluation of the Newcastle-Ottawa scale for the assessment of the quality of nonrandomized studies in meta-analyses. Europ J Epidemiol 2010; 25: 603-605

[13] Glenny A, Altman D, Song F et al. Indirect comparisons of competing interventions. Health Technol Assess 2005; 9: 1-134

[14] de Franchis R. Revising consensus in portal hypertension: report of the Baveno $V$ consensus workshop on methodology of diagnosis and therapy in portal hypertension. J Hepatol 2010; 53: 762-768

[15] DerSimonian R, Laird N. Meta-analysis in clinical trials. Controlled Clin Trials 1986; 7: 177-188

[16] Sutton AJ, Abrams KR, Jones DR et al. Methods for meta-analysis in medical research. John Wiley \& Sons Ltd New York 2000: 205-228

[17] Higgins J, Thompson SG, Spiegelhalter DJ. A re-evaluation of randomeffects meta-analysis. Stat Med 2009; 172: 137-159

[18] Riley RD, Higgins JP, Deeks JJ. Interpretation of random effects metaanalyses. BMJ 2011; 342: d549

[19] Mohan BP, Adler DG. Heterogeneity in systematic review and metaanalysis: how to read between the numbers. Gastrointest Endosc 2019; 89: 902-903

[20] Kanwal F, White D. Systematic reviews and meta-analyses in clinical gastroenterology and hepatology. Clin Gastroenterol Hepatol 2012; 11: $1184-1186$
[21] Higgins JP, Thompson SG, Deeks J] et al. Measuring inconsistency in meta-analyses. BMJ 2003; 327: 557

[22] Guyatt GH, Oxman AD, Kunz R et al. GRADE guidelines: 7. Rating the quality of evidence\&\#x2014;inconsistency. J Clin Epidemiol 2011; 64: 1294-1302

[23] Easterbrook PJ, Gopalan R, Berlin JA et al. Publication bias in clinical research. The Lancet 1991; 8746: 867-872

[24] Duval S, Tweedie R. Trim and fill: a simple funnel-plot-based method of testing and adjusting for publication bias in meta-analysis. Biometrics 2000; 56: 455-463

[25] Rothstein HR, Sutton AJ, Borenstein M. Publication bias in meta-analysis: Prevention, assessment and adjustments. John Wiley \& Sons; 2006

[26] Dechene A, El Fouly AH, Bechmann LP et al. Acute management of refractory variceal bleeding in liver cirrhosis by self-expanding metal stents. Digestion 2012; 85: 185-191

[27] Drastich P, Brezina J, Sperl J et al. Treatment of uncontrollable acute variceal bleeding with self-expanding metal stent: A single center experience. Gastroenterology 2016; 1: S339

[28] Escorsell A, Pavel O, Cardenas A et al. Esophageal balloon tamponade versus esophageal stent in controlling acute refractory variceal bleeding: A multicenter randomized, controlled trial. Hepatology 2016; 63: 1957-1967

[29] Fierz FC, Kistler W, Stenz V et al. Treatment of esophageal variceal hemorrhage with self-expanding metal stents as a rescue maneuver in a swiss multicentric cohort. Case Rep 2013; 7: 97-105

[30] Mishin I, Zastavnitsky G, Ghidirim G et al. Self-expanding metal stents: A new hemostasis method for bleeding esophageal varices. Hepatol Int 2013; 1: S540

[31] Muller M, Seufferlein T, Perkhofer L et al. Self-expandable metal stents for persisting esophageal variceal bleeding after band ligation or injection-therapy: a retrospective study. PLoS ONE 2015; 10: e0126525

[32] Wright G, Lewis H, Hogan B et al. A self-expanding metal stent for complicated variceal hemorrhage: experience at a single center. Gastrointest Endosc 2010; 71: 71-78

[33] Zakaria MS, Hamza IM, Mohey MA et al. The first Egyptian experience using new self-expandable metal stents in acute esophageal variceal bleeding: pilot study. Saudi J 2013; 19: 177-181

[34] Zehetner J, Shamiyeh A, Wayand W et al. Results of a new method to stop acute bleeding from esophageal varices: implantation of a selfexpanding stent. Surg Endosc 2008; 22: 2149-2152

[35] Cello JP, Ring EJ, Olcott EW et al. Endoscopic sclerotherapy compared with percutaneous transjugular intrahepatic portosystemic shunt after initial sclerotherapy in patients with acute variceal hemorrhage. A randomized, controlled trial. Ann Intern Med 1997; 126: 858-865

[36] Garcia-Pagan JC, Di Pascoli M, Caca K et al. Use of early-TIPS for highrisk variceal bleeding: results of a post-RCT surveillance study. J Hepatol 2013; 58: 45-50

[37] Monescillo A, Martinez-Lagares F, Ruiz-del-Arbol L et al. Influence of portal hypertension and its early decompression by TIPS placement on the outcome of variceal bleeding. Hepatology 2004; 40: 793-801

[38] Popovic P, Stabuc B, Skok P et al. Transjugular intrahepatic portosystemic shunt versus endoscopic sclerotherapy in the elective treatment of recurrent variceal bleeding. J Int Med Res 2010; 38: 11211133

[39] Rudler M, Cluzel P, Corvec TL et al. Early-TIPSS placement prevents rebleeding in high-risk patients with variceal bleeding, without improving survival. Aliment Pharmacol Ther 2014; 40: 1074-8010

[40] Shi Y, Tian X, Hu J et al. Efficacy of transjugular intrahepatic portosystemic shunt with adjunctive embolotherapy with cyanoacrylate for esophageal variceal bleeding. Dig Dis Sci 2014; 59: 2325-2332 
[41] Xue H, Zhang M, Pang JX et al. Transjugular intrahepatic portosystemic shunt vs endoscopic therapy in preventing variceal rebleeding. World J Gastroenterol 2012; 18: 7341-7347

[42] Orloff M], Vaida F, Haynes KS et al. Randomized controlled trial of emergency transjugular intrahepatic portosystemic shunt versus emergency portacaval shunt treatment of acute bleeding esophageal varices in cirrhosis. J Gastrointest Surg 2012; 16: 2094-2111

[43] Hogan B, Patch D, Burroughs A et al. Use of the sx-ella self-expanding mesh metal stent in the management of complex variceal haemorrhage: initial experience in a single centre. J Hepatol 2009; 50: S86S87

[44] Hubmann R, Bodlaj G, Czompo M et al. The use of self-expanding metal stents to treat acute esophageal variceal bleeding. Endoscopy 2006; 38: 896-901
[45] Puhan MA, Schunemann HJ, Murad MH et al. A GRADE Working Group approach for rating the quality of treatment effect estimates from network meta-analysis. BMJ 2014; 349: g5630

[46] McCarty TR, Njei B. Self-expanding metal stents for acute refractory esophageal variceal bleeding: A systematic review and meta-analysis. Dig 2016; 28: 539-547

[47] Marot A, Trepo E, Doerig C et al. Systematic review with meta-analysis: self-expanding metal stents in patients with cirrhosis and severe or refractory oesophageal variceal bleeding. Aliment Pharmacol Ther 2015; 42: 1250-1260

[48] Shao XD, Qi XS, Guo XZ. Esophageal stent for refractory variceal bleeding: a systemic review and meta-analysis. BioMed Research Int 2016; 2016: 4054513

[49] Qi X, Jia J, Bai M et al. Transjugular Intrahepatic Portosystemic Shunt for Acute Variceal Bleeding: A Meta-analysis. J Clin Gastroenterol 2015; 49: 495-505 